\title{
Inter-individual performance differences in younger and older adults differentially relate to amplitude modulations and phase stability of oscillations controlling working memory contents
}

\author{
Markus Werkle-Bergner ${ }^{\mathrm{a}, *}$, Roman Freunberger ${ }^{\mathrm{a}, \mathrm{b}}$, Myriam C. Sander ${ }^{\mathrm{a}}$, \\ Ulman Lindenberger ${ }^{\mathrm{a}}$, Wolfgang Klimesch ${ }^{\mathrm{b}}$ \\ a Center for Lifespan Psychology, Max Planck Institute for Human Development, Berlin, Germany \\ b Department of Physiological Psychology, University of Salzburg, Salzburg, Austria
}

\section{A R T I C L E I N F O}

\section{Article history:}

Received 12 September 2011

Revised 22 November 2011

Accepted 23 November 2011

Available online 8 December 2011

\section{Keywords:}

Aging

Alpha activity

EEG

Oscillations

Phase-stability

Visual working memory

\begin{abstract}
A B S T R A C T
Efficient encoding of relevant information and suppression of irrelevant information influence working memory (WM) performance, which is limited and declines in adulthood. A cued Sternberg WM task and electroencephalographic recordings (EEG) were used to investigate encoding and control operations in response to to-be-remembered (REM) and not-to-be-remembered (NREM) stimuli in younger and older adults. Younger and older adults selectively remembered REM items in a final recognition memory test. During early stages of stimulus processing, inter-trial phase stability was higher for REM than for NREM items in younger and older adults, presumably reflecting preferential encoding of REM items. At later stages, the oscillatory power of oscillations in the alpha/beta frequency range was higher for NREM than for REM, presumably reflecting the inhibitory top-down suppression of task-irrelevant information. Early phase stability was selectively related to working memory performance in younger adults and high-functioning older adults. The results of this study reveal the differential contributions of low-level feature binding and strategic control components to adult age differences in WM, and show that older adults with more youth-like processing dynamics tend to achieve higher levels of performance.
\end{abstract}

(c) 2011 Elsevier Inc. All rights reserved.

\section{Introduction}

The capacity of working memory (WM), our ability to shortly maintain and manipulate relevant information, is constrained (Luck and Vogel, 1997; Miller, 1956) and shows large variation within and between persons (Eng et al., 2005; Vogel and Awh, 2008). One important factor contributing to interindividual differences in the WM performance range is the efficient control of WM contents (Freunberger et al., 2009; Gazzaley et al., 2005, 2008; Sauseng et al., 2009; Vogel et al., 2005), in particular, when both relevant and irrelevant information is present in the environment.

Recent evidence suggests that the inhibition of irrelevant information is closely related to the power of oscillatory brain activity in the alpha-frequency range (i.e., 7-14 Hz) (Freunberger et al., 2009, 2011; for a recent review, see Klimesch et al., 2007; Palva and Palva, 2007; Romei et al., 2008; Thut et al., 2006; van Dijk et al., 2010). For example, by combining EEG and repetitive transcranial magnetic stimulation (rTMS), Sauseng et al. (2009) recently found that the amplitude of lateralized alpha oscillations co-varies with the amount of

\footnotetext{
* Corresponding author at: Center for Lifespan Psychology, Max Planck Institute for Human Development, Lentzeallee 94, 14195 Berlin, Germany. Fax: +49308249939. E-mail address: werkle@mpib-berlin.mpg.de (M. Werkle-Bergner).
}

irrelevant information and predicts individual WM capacity. More importantly, the application of rTMS (at alpha frequencies) over cortical sites processing irrelevant information led to increments in WM performance. Relatedly, when participants successfully ignore taskirrelevant stimuli, alpha amplitudes increase already prior to stimulus-onset (Freunberger et al., 2009) (Fig. 1).

In addition to the role of oscillatory power for inhibition processes, the synchronicity of neural oscillations provides a mechanism for the timing of neuronal information processing and the successful formation of integrated representations, i.e., binding (Fries, 2005; Klimesch et al., 2007; Singer and Gray, 1995; Von der Malsburg, 1981). For example, increased inter-trial phase stability for relevant compared to irrelevant information reflects a restructuring of neuronal information processing in the service of perceptual encoding (Hanslmayr et al., 2005; Mathewson et al., 2009). In a similar vein, Klimesch et al. (2011) argue that alpha phase locking in response to stimulus presentations reflects the successful encoding of global stimulus features, i.e., feature binding.

With advancing adult age, WM performance declines (Cowan et al., 2006; Park and Payer, 2006). Prominent aging theories posit decrements in control processes as one of the key determinants of general cognitive decline (Baltes and Lindenberger, 1997; Hasher and Zacks, 1988; Salthouse, 1996). This theoretical proposition is supported by recent studies that observed a selective age deficit in the 


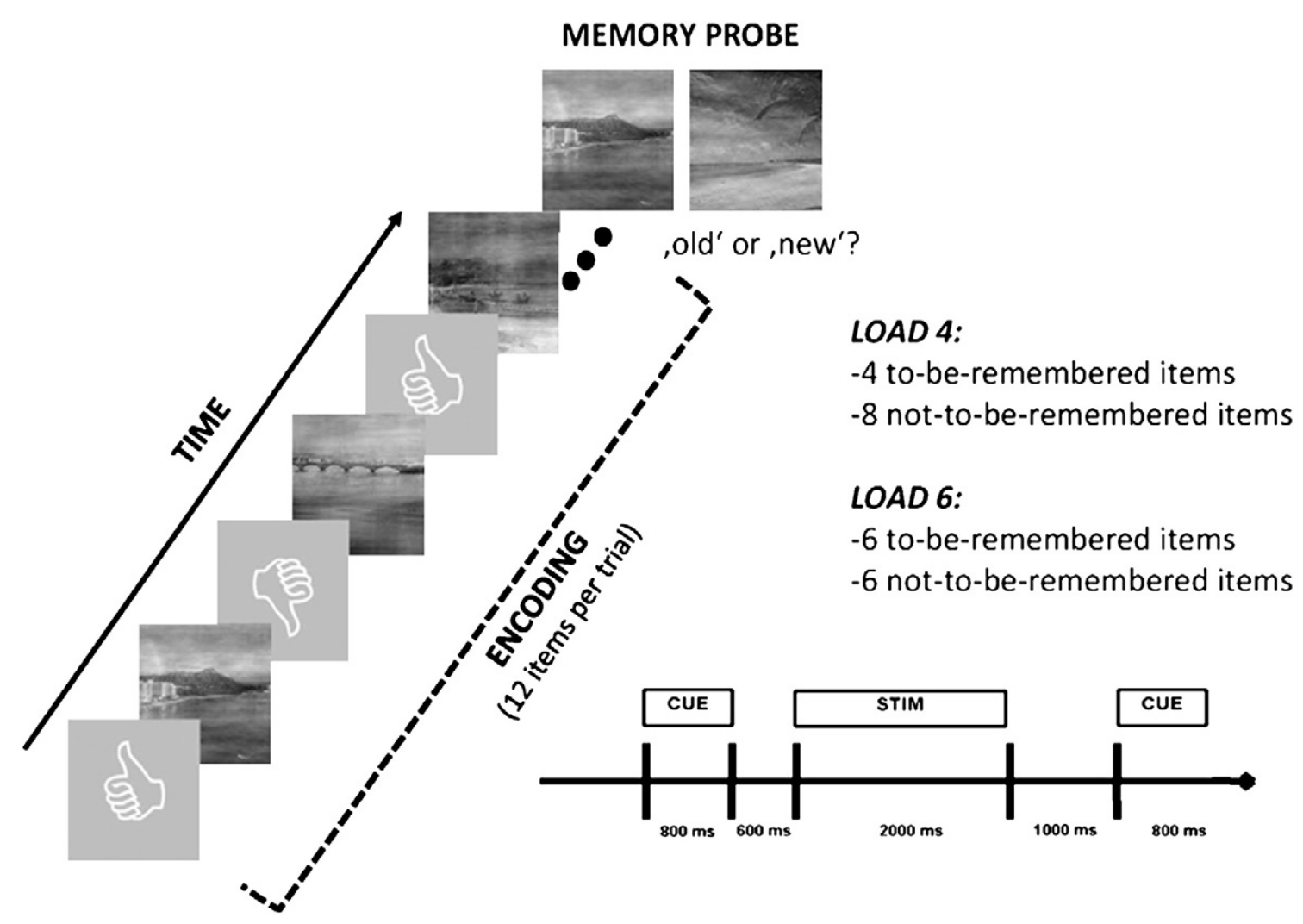

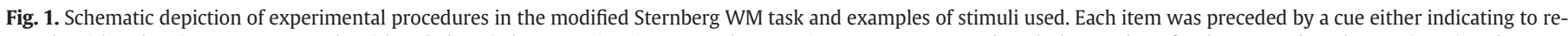

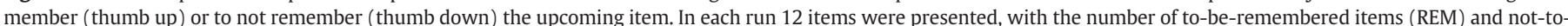

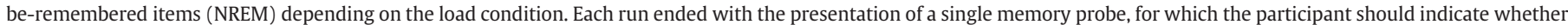
it was part of the memory set ('old') or not ('new').

suppression of irrelevant information in particular during early stages of WM encoding (Gazzaley et al., 2005, 2008). These studies suggest that inhibitory control of WM contents may not be disabled completely in older adults, but rather delayed in time (Jost et al., 2011; Zanto et al., 2010). In addition, the robustness of inhibitory mechanisms depends on the task difficulty and the individual performance level. A recent study by Sander et al. (2012) demonstrated that late inhibitory processes reflected in lateralized alpha power differences between relevant and irrelevant information are less robust in older adults and break down when WM capacity is overtaxed.

How the interaction between early and late WM control processes is affected in advancing age is still an open question. Nevertheless, the above cited findings suggest that, age deficits in early control mechanisms may impair later processing stages by increasing the demands on late selection processes (Velanova et al., 2007) and this effect may be exaggerated when individual processing limits are reached (Sander et al., 2012).

Here, we examine whether phase-locked and non-phase-locked oscillatory brain activity differentially contributes to age differences in controlling the contents of WM, by assessing task related modulations of inter-trial phase-stability and oscillatory power, respectively. Towards this end, younger adults and older adults performed a cued version of the Sternberg WM paradigm with variable set sizes (Freunberger et al., 2009; Sternberg, 1966). In this task, participants were informed prior to stimulus presentation whether they should memorize or ignore the upcoming stimulus. This procedure allowed to dissociate neuronal processes related to the encoding of relevant information from control mechanisms in the service of suppression of irrelevant information. A first set of analyses therefore used a data-driven approach to identify reliable effect patterns that dissociate the processing of relevant versus irrelevant information in younger and older adults. To anticipate the results, we observed similar neural mechanisms in both age groups. To test for the possibility that younger and older adults differentially recruit a subset of processes to support performance, we conducted neuro-behavioral correlation analyses for the identified effects in order to reveal their contribution to inter-individual differences in WM performance.

At the same time, individual differences in WM performance increase with advancing age (Lindenberger et al., 2008). This increase in performance heterogeneity is often ignored when younger and older adults are compared in neural correlates of WM. However, recent reports from functional magnetic resonance imaging studies (fMRI) suggest a dependence of fMRI brain activation patterns on the performance level in WM tasks (Nagel et al., 2009; SchneiderGarces et al., 2010). Therefore, we checked the age groups in the present study with regard to performance homogeneity in the WM task. To foreshadow the results, we found evidence for a unimodal performance distribution in younger, but a bimodal distribution in older adults, supporting the assumption of homogeneous performance levels in younger, but not in older adults. A second set of analyses therefore took the performance level of older adults (high versus low performers) into account when comparing the oscillatory EEG correlates of WM processes.

\section{Materials and methods}

\subsection{Participants}

The effective sample comprised 20 younger adults (YA; mean age 23.9 years, range 21.6 to 25.8 years, 11 women), and 22 older adults (OA; mean age 72.9 years, range 70.3 to 76 years, 8 women). Descriptive sample characteristics and a summary of covariate measures are presented in Table 1 . The participants were recruited from the participant pool of the Max Planck Institute for Human Development, 
Table 1

Descriptive summary of covariate measures.

\begin{tabular}{|c|c|c|}
\hline \multirow[t]{2}{*}{ Measure } & $\begin{array}{l}\text { Young adults } \\
(n=19)\end{array}$ & $\begin{array}{l}\text { Older adults } \\
(\mathrm{n}=21)\end{array}$ \\
\hline & $M(S D)$ & $M(S D)$ \\
\hline Age & $23.93(1.39)$ & $72.90(1.68)$ \\
\hline Digit symbol substitution test & $76.16(10.74)$ & $51(8.96)$ \\
\hline Vocabulary & $24.11(3.68)$ & $28.82(2.84)$ \\
\hline Close vision & $0.90(.09)$ & $0.38(.16)$ \\
\hline Far vision & $1.39(.49)$ & $0.80(.47)$ \\
\hline
\end{tabular}

Note: Independent samples t-tests (assuming unequal variance and sample size) reveal reliable differences between age groups in all covariate measures (with t-values ranging from 3.92 to 12.64; all $\mathrm{p}<0.0004$ ). The high- and low-performing groups of older adults did not differ in any of the measures (all $\mathrm{t}<1.38$; all $\mathrm{p}>.18$ ).

Berlin, Germany (MPIB). All participants provided written informed consent according to institutional guidelines of the ethics committee of the MPIB. All participants were right-handed, as assessed with the Edinburgh Handedness Inventory (Oldfield, 1971), reported to be in good health with no known history of neurological or psychiatric diseases, and were paid for participation. In addition to participants included in the effective sample, data from one younger adult, and four older adults were also recorded. However, these data had to be discarded from further analyses after artifact rejection due to excessive muscle noise or technically unsatisfactory recordings. In addition, one younger participant did not complete the final recognition test due to scheduling issues (data from this participant is included in the analysis of the WM task).

\subsection{Experimental procedures}

Prior to electrode placement, participants filled out a demographic and health-related questionnaire. Visual acuity and two marker tests of fluid and crystallized intelligence were assessed as well.

Visual acuity was measured in Snellen decimal units at two different distances using a standard table with Landolt rings (Geigy, 1977). Close visual acuity was measured separately for the left and the right eye at a distance of $30 \mathrm{~cm}$. Distance visual acuity was assessed binocularly at a distance of $5 \mathrm{~m}$ to the participant. All measures were taken with the best optical correction (i.e., glasses) available to the participant because the experimental procedures were carried out in the same mode.

Two cognitive tests were used to document the age typicality of the sample. Perceptual speed as a broad fluid marker was assessed with the Digit Symbol Substitution Test (Wechsler, 1958), and verbal knowledge as a broad crystallized marker was assessed with the Spot-a-Word test (adapted from Lehrl, 1977; see Lindenberger et al., 1993).

After electrode placement, participants were seated comfortably in a dimly lit as well as electromagnetically and acoustically shielded room. The EEG measurement started with a 6-minute relaxation phase (resting-state EEG: $3 \mathrm{~min}$ with eyes closed and $3 \mathrm{~min}$ with eyes open). Afterwards, the participants performed one practice block and six experimental blocks (with eight trials per block) of the WM task described below.

After the EEG measurement, participants had a short break of 15-20 min, and were then informed about a surprise recognition memory test for the images from the WM phase (described in detail below). The whole session lasted approximately $3.5 \mathrm{~h}$.

\subsection{Stimuli}

Scene stimuli were taken from a publicly available database (Fei-Fei and Perona, 2005). Each stimulus was depicted in gray-scale ( 8 bit/ pixel) and had a size of $256 \times 256$ pixel. All images were equated for luminance and contrast, and had identical magnitude spectra (i.e., the average magnitude spectrum of all images used). The average magnitude spectrum was combined with the corresponding phase spectrum of each single image (see Philiastides and Sajda, 2006 for similar procedures). Stimuli were presented on a gray background via a Liquid Crystal Monitor (LCD, refresh rate: $60 \mathrm{~Hz}$ ). The stimuli subtended approximately $4^{\circ}$ by $4^{\circ}$ of visual angle from a viewing distance of $70 \mathrm{~cm}$. Stimulus-presentation and recording of behavioral responses were controlled with the E-Prime v1.2 software (Psychology Software Tools, Inc., Pittsburgh, PA, USA).

\subsection{Modified Sternberg WM task}

During WM assessment, each trial began with a screen presented for $1000 \mathrm{~ms}$ indicating the start of the next trial. Afterwards, a cue was shown for $800 \mathrm{~ms}$. Cues indicated whether the following scene stimulus should be part of the memory set or should be ignored. The cues were $100 \%$ valid. After a blank screen interval $(600 \mathrm{~ms})$ a scene stimulus was shown for $2000 \mathrm{~ms}$, which was again followed by a blank interval $(1000 \mathrm{~ms})$. Within each trial, 12 stimuli were presented in total. WM load was manipulated by varying the number of to-be-remembered (REM) and not-to-be-remembered (NREM) cues. Accordingly, a LOAD 4 condition contained four REM and eight NREM stimuli, while the LOAD 6 condition contained six REM and six NREM items. Immediately after the last scene stimulus of a given trial, a blank screen interval of 2000 ms marked the retention delay. This was followed by a warning screen $(1000 \mathrm{~ms})$, after which one memory probe was shown for up to $5000 \mathrm{~ms}$, or until the participant's response.

After practice, participants performed six experimental blocks, with eight trials per block. Each experimental block contained four LOAD4 and four LOAD6 trials. For half of the trials in each block and load-condition, the probe stimulus had been part of the memory set. For the other half of the trials, the probe stimulus was a completely new item. Responses were collected with a custom build response pad and the response assignment to the left and right index finger was balanced across participants. The start of the following trial was self-paced by the participant.

\subsection{Final recognition memory test}

For the unexpected final recognition test, 40 REM and 56 NREM stimuli were selected from the third and fourth experimental block of the WM task (the unequal numbers are due to the number of available stimuli in the third and fourth block). These old scene stimuli were intermixed with 91 new items, not seen before. The two first and two last blocks of the WM task were discarded to prevent primacy and recency effects on later recognition performance. To assure that none of the old items in the recognition test had been presented more than once before, probe items were not included. Participants were instructed to respond 'old' to all items seen during the WM assessment, that is, to both REM and NREM items. Each trial started with a fixation cross for $1000 \mathrm{~ms}$, followed by presentation of the recognition probe $(3000 \mathrm{~ms})$. After indicating their old/new judgment, participants were asked for their confidence on a three-point scale from 'sure' (in German 'sicher') to unsure ('unsicher').

\subsection{EEG recordings and preprocessing}

EEG was recorded continuously (BrainAmp amplifiers, Brain Products $\mathrm{GmbH}$, Gilching, Germany) from $60 \mathrm{Ag} / \mathrm{Ag}-\mathrm{Cl}$ electrodes embedded in an elastic cap (EASYCAP GmbH, Herrsching, Germany). To monitor eye movements, three additional electrodes were placed at the outer canthi (horizontal EOG), and below the left eye (vertical EOG). During recording, all electrodes were referenced to the right mastoid electrode, the left mastoid electrode was recorded as an 
additional channel, and ground was placed at location AFz. Electrode impedances were maintained below $5 \mathrm{k} \Omega$ before recordings. The EEG was recorded with a pass-band of 0.1 to $250 \mathrm{~Hz}$ and digitized with a sampling rate of $1000 \mathrm{~Hz}$ for later off-line analyses.

Preprocessing comprised the following steps: First, after rereferencing to linked mastoids, the Laplacian current source density was calculated for all EEG channels (order of splines: 4; maximum degree of Legendre polynomials: 10; lambda: $1 \mathrm{e}-5$ ) in order to attenuate effects due to volume conduction. Second, the data from the WM encoding phase were segmented from $-2 \mathrm{~s}$ (600 ms prior to cueonset) until $2 \mathrm{~s}$ after stimulus onset and the mean across the entire $4 \mathrm{~s}$ epoch was subtracted to control for drifts. Note that no further baseline correction was applied in any of the later processing steps. Third, trials from all experimental conditions were combined (within subjects) and automatically screened for extreme or untypical eye/ muscle artifacts by means of kurtosis and frequency measures (Delorme et al., 2007) as follows: We computed the kurtosis and Fourier-spectra of each single trial. Afterwards, the distribution of kurtosis values, as well as the mean power in low $(0.5 \mathrm{~Hz}$ to $5 \mathrm{~Hz})$ and high frequency ranges $(15 \mathrm{~Hz}$ to $100 \mathrm{~Hz}$ ) was determined. Trials were discarded from further analyses if the respective kurtosis/ power value exceeded the $99 \%$ confidence interval at any electrode location. The remaining trials were concatenated. Fourth, an independent component analysis (ICA; Delorme et al., 2007; Jung et al., 2000) was performed. Each independent component (IC) was visually evaluated based on their topographical profile, time course, and frequency characteristics. ICs reflecting eye or muscle activity were discarded, and the remaining ICs were used for backprojection into sensorspace. All further analyses were carried out in sensor-space based on the backprojection of non-artifact ICs.

\section{Data analysis}

\subsection{Analysis of EEG data}

Time- and frequency-domain analyses of the EEG data were carried out using the FieldTrip software package (developed at the F.C. Donders Centre for Cognitive Neuroimaging, Nijmegen, The Netherlands; http://www2.ru.nl/fcdonders/fieldtrip/) and custom-made routines operated in MATLAB 7.4 (The MathWorks Inc., Natick, MA, USA).

To extract oscillatory power and phase from $4 \mathrm{~Hz}$ to $30 \mathrm{~Hz}$ in steps of $1 \mathrm{~Hz}$, the amplitude time-series of each single trial at each electrode was convolved with a set of Morlet-wavelets of seven cycles length. The time-varying power of the signal for each center frequency is given by the squared norm of the respective complex-valued wavelet-coefficients. The time-varying phase is given by computing the inverse tangents of the ratio of the real and imaginary parts of the wavelet coefficients (e.g., Tallon-Baudry et al., 1998; WerkleBergner et al., 2009).

The general analysis strategy for the time-frequency decomposed data followed a summary statistics approach as it is common for functional magnetic resonance imaging (fMRI) data (Holmes and Friston, 1998; Mumford and Poldrack, 2007). For the first-level analysis, we quantified the main effect of interest, i.e., the difference between to be remembered and not to be remembered items, within subjects across trials by means of $t$-tests. The resulting statistical maps were further subjected to second-level between subjects analyses by comparing the mean effect across subjects (expressed as $z$-values) against zero. To control for multiple comparisons, the resulting groupstatistical maps were thresholded by means of a false-discovery rate procedure (FDR, Benjamini and Hochberg, 1995; Nichols and Hayasaka, 2003) assuming an overall significance level of $p<0.01$. Note that therefore Figs. 4 and 7 directly represent the statistical results, similar to statistical parameter maps in fMRI analyses. The specific details for the analysis of power and phase information will be outlined below.

For analyses of oscillatory power, the difference between NREM and REM items was quantified within subject by means of independent sample $t$-test (assuming unequal number of trials and unequal variance) for each time-frequency point at each electrode. The resulting $t$-values were converted to $z$-scores (Hughett, 2007), and pooled across posterior channels (i.e., P1/2, P3/4, P5/6, P7/8, Pz, PO3/4, $\mathrm{PO} 7 / 8, \mathrm{POz}, 01 / 2, \mathrm{Oz}$ ). The choice for this cluster of electrodes was motivated by previous studies reporting oscillatory correlates of WM-control mechanisms to be maximal over posterior regions (e.g., Freunberger et al., 2009; Sander et al., 2012). The final z-values represent the normalized difference between REM and NREM items (van Dijk et al., 2010). Variance estimates for each time-frequency point were derived with a leave-one-out jackknife procedure (Bauer et al., 2006). Afterwards, group analyses were conducted separately for younger and older adults. At each time-frequency point, we tested the mean $z$-score across subjects against zero by means of a $z$-test, assuming no difference between NREM and REM items on the group level. From the resulting statistical maps, masks were created to identify regions with statistically reliable differences. To control for multiple comparisons, a FDR was applied assuming an overall significance level of $p<0.01$.

To quantify the phase stability of ongoing oscillations across trials (= inter-trial phase stability), the phase-locking index (e.g., Lachaux et al., 1999) was computed for each subject, electrode, and timefrequency point, separately for REM and NREM items. Again, variance estimates were derived with a jackknife procedure (for details see, Werkle-Bergner et al., 2009). Afterwards, the same statistical procedures (within subject $t$-test, conversion to $z$-scores, pooling, group analysis, thresholding etc.) were performed as described for the analyses of oscillatory power.

This procedure allowed for a data-driven definition of timefrequency regions of interest (TF-ROIs), based on the resulting statistical maps separately for each age group for power (Fig. 4) and intertrial phase-stability (Fig. 7) analyses. Note that these tf-ROIs themselves represent already statistically significant results, similar to statistical parameter maps in fMRI analyses.

To extract a mean z-score for each subject within the TF-ROI, the respective z-score maps (pooled over posterior electrodes) were masked with the group-level effect. In each TF-ROI, the mean zvalue for each subject was then calculated by averaging timefrequency points that revealed a reliable difference at the group level. Note that this procedure guarantees that all time-frequency points contributing to the single-subject estimates do indeed show a statistically reliable, i.e., $\mathrm{p}<.05$, difference at the group level. These individual $\mathrm{z}$-scores were then used for age group comparisons and neuro-behavioral correlation analyses.

\subsection{Analysis of behavioral data}

To compare performance in the WM task between age groups the percentage of correct responses as well as the median response latencies within subjects was computed separately for each of the two load levels. Afterwards, mixed measures analyses of variance (ANOVA) were conducted with age group (YA vs. OA) as between-subjects factor and load (four items vs. six items) as within-subjects factor, separately for percentage correct and RT.

To quantify the performance in the unexpected final recognition test, hit rates (HR; i.e., correct 'old' responses to items shown during the WM test) and false alarm rates (FAR; i.e., incorrect 'old' responses to new items) were computed for each subject. Corrected recognition scores (i.e., Pr-values; Snodgrass and Corwin, 1988) were calculated as HR minus FAR, and analyzed with a mixed measures ANOVA with age group (young adults vs. older adults) as between-subjects and item type (REM vs. NREM) as within-subjects factor. 
Omnibus ANOVAs were followed up by contrasts when appropriate. The alpha level for all statistical analyses was set to $\alpha=.05$, with Bonferroni adjustments when needed. For reliable effects and for trends $(.1>p>.01)$, effect sizes are indicated by reporting partial eta-squared $\left(\eta_{\mathrm{p}}^{2}\right.$; (Hullett and Levine, 2003) or Cohen's $d$ (Cohen, 1988). All analyses involving behavioral data were computed with $R$ (http://www.r-project.org/).

Recent reports suggest that age differences in neural activation patterns in WM tasks may depend on the individual performance level (Nagel et al., 2009; Schneider-Garces et al., 2010). In addition to mean level comparisons of performance, we therefore checked the homogeneity of our YA and OA samples (see Fig. 2B). To quantify age-group specific between-person differences in WM performance, we compared the distribution of percentage correct scores across load conditions within age groups for deviations from unimodality by means of the dip test (Hartigan and Hartigan, 1985). To test for statistical significance, 1000 random samples from a uniform random distribution were drawn, with the same sample size as the groups of younger and older adults, respectively. For each random sample, the respective value of the dip statistic was calculated. The actual values for younger and older adults were compared against the bootstrapped distributions of dip values from the uniform random distribution.

\subsection{Analysis of neurobehavioral correlations}

To test whether task dependent modulations in oscillation amplitude and/or phase-stability predict WM performance, we conducted neurobehavioral correlation analyses between the percentage of correct responses in the WM task and the normalized differences
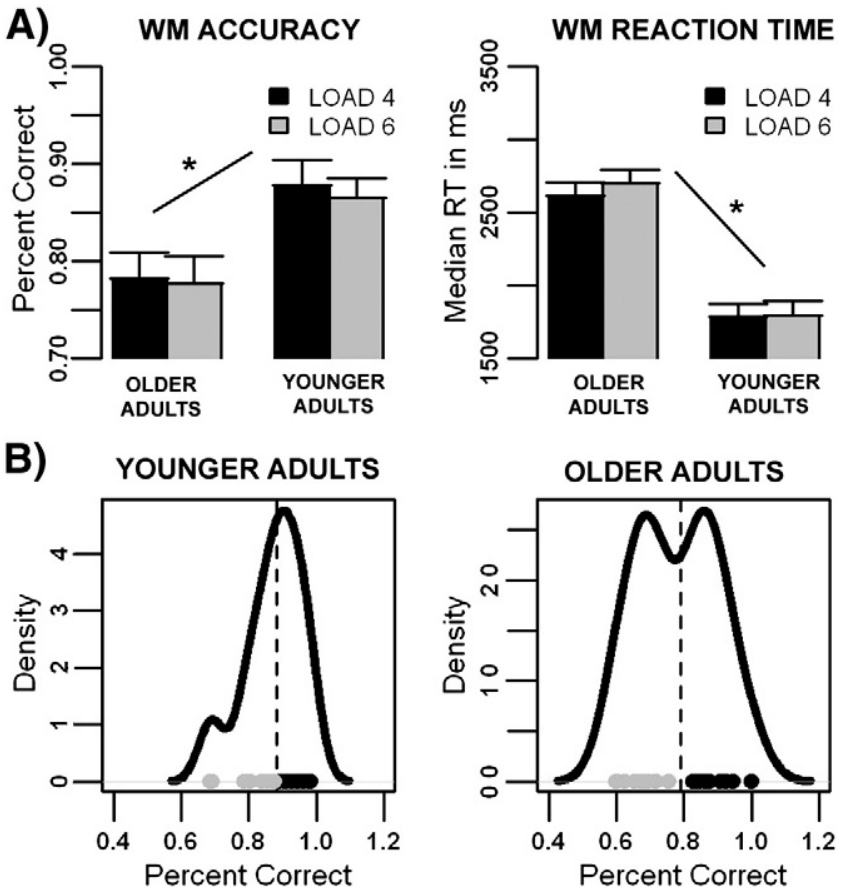

Fig. 2. Summary of behavioral results in the WM task. (A) Mean percentage of correct responses (left-hand side of panel A, "WM Accuracy") and mean of the median response latencies (right-hand side of panel A, "WM Reaction Time"), for older and younger adults, separately for LOAD 4 (black) and LOAD 6 (gray) conditions. Note: Error bars in panel $(A)$ indicate the standard error of the mean. Reliable effects $(\mathrm{p}<.05)$ are marked my asterisks. (B) Inter-individual differences in WM accuracy (percentage of correct responses averaged across LOAD 4 and LOAD 6 conditions) reveal a unimodal distribution (black curve) in younger adults (left-hand side of panel C) and a bimodal distribution in older adults (right-hand side of panel C). Each dot represents a single participant. The broken vertical line marks the group-specific median. Participants below or above median performance (group-specific) are marked in gray and black, respectively. (expressed in $z$-scores) in oscillatory power (NREM minus REM) and indicators of phase-stability (REM minus NREM).

To control for possible effects of outliers that are likely to bias the analyses with small samples, we conducted a bootstrap analysis on the neurobehavioral correlations, separately for the different participant groups. For this set of analyses, we drew 10,000 random samples with replacement. For each bootstrap replication, we calculated the correlation coefficient. Afterwards, we determined the 95\% confidence interval $\left(\mathrm{Cl}_{\text {boot }}\right)$ around the mean correlation, by identifying the lower and upper 2.5\%-values of the sorted bootstrapcorrelations. Correlations were regarded reliable, if the bootstrapped 95\% CI did not include zero, which corresponds to a significance level of $\alpha<.05$.

\section{Results}

\subsection{Behavioral performance}

With regard to WM performance (see Fig. 2), mixed measures ANOVAs revealed a main effect of age group for correct responses, $F(1,40)=8.3, p<.05, \eta^{2}=.17$, and response latencies, $F(1,40)=48.3$, $p<.05, \eta^{2}=.55$. YA $(M=.87, S E=.02)$ showed higher performance than $\mathrm{OA}(M=.78, S E=.03)$ as well as shorter response latencies (YA: $M=1788 \mathrm{~ms}, S E=91.9 ; \mathrm{OA}: M=2654 \mathrm{~ms}, S E=88.2$ ). Neither the load main effects (LOAD 4 vs. LOAD 6 ), nor the age group $\times$ load interactions reached significance, all $F s<1.8$, all $p s>.18$.

The omnibus ANOVA of the final recognition test (see Fig. 3 ) revealed a main effect of item type (REM vs. NREM), $F(1,39)=95.3, p<.05$, $\eta^{2}=.71$, and an item type $\mathrm{x}$ age group interaction, $F(1,39)=8.7$, $p<.05, \eta^{2}=.18$. Post-hoc $t$-tests confirmed that in both age groups REM items were recognized reliably above chance, YA: $t(18)=7.7$, $p<.0125$; OA: $t(21)=7.1, p<.0125$. In contrast, recognition performance for NREM items was at chance level in both age groups, YA: $t(18)=1.1, p>.1$; OA: $t(21)=1.5, p>.1$. Thus, apparently, participants from both age groups actively suppressed the processing of NREM items during the WM phase while trying to efficiently encode the relevant information from REM items. In addition, younger adults showed higher recognition performance for REM items than older adults, Welch's test: $t(31)=2.5, p<.05, d=.88$.

In addition to mean performance level differences between younger and older adults, we observed larger performance heterogeneity in our sample of older relative to younger adults. The analysis of age group homogeneity by means of the dip test revealed $p$-values of $p>.80$ and $p<.05$ for younger and older adults, respectively, supporting the assumption of unimodality for younger but not for older adults. Put differently, this result suggests the presence of two distinct groups of $\mathrm{OA}$ within our participant sample. Close inspection of Fig. 2B reveals that the low (OA-L) and high $(\mathrm{OA}-\mathrm{H})$ performing groups are indeed clearly separated by the median (broken vertical

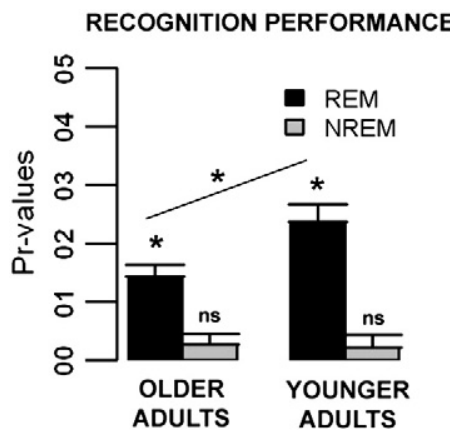

Fig. 3. Summary of recognition memory performance. Corrected recognition scores, i.e., Pr-values (computed as HR minus FAR), for older and younger adults, separately for REM (black) and NREM (gray) items. Note: Error bars indicate the standard error of the mean. Reliable effects $(\mathrm{p}<.05)$ are marked by asterisks. 
line in Fig. 2B), i.e., a traditional median split would result in exactly the same division of older participants. In fact, WM performance was reliably higher in OA-H $(M=0.88, S E=.02)$ compared to OA-L $(M=0.68, S E=.02), t(19.8)=9.3, p<.05$. Although not originally intended as a design factor, for some analyses of the EEG measures, we will split the group of older adults in order to highlight the role of performance heterogeneity for the interpretation of age-group differences in oscillatory EEG measures.

\subsection{Oscillatory power}

With regard to oscillatory power, four effect patterns were found to reliably dissociate the processing of NREM from REM items in both younger and older adults during WM encoding (see Fig. 4), with higher oscillatory power for NREM than for REM items. First, already in response to the instruction cue, NREM items elicited higher power (with a peak in the alpha frequency range) than REM items (CUE effect; $1000 \mathrm{~ms}$ to $-500 \mathrm{~ms} ; 7 \mathrm{~Hz}$ to $12 \mathrm{~Hz}$ ). Second, also at stimulus onset, alpha power was higher for NREM items than for REM items (STIM effect; - $200 \mathrm{~ms}$ to $200 \mathrm{~ms} ; 7 \mathrm{~Hz}$ to $12 \mathrm{~Hz}$ ). In addition, two additional effects representing reliable differences in oscillatory power between NREM and REM items occurred during later phases of stimulus presentation $(600 \mathrm{~ms}$ to $1700 \mathrm{~ms}$ ) that could be divided into a lower component in the alpha frequency range (LATE LOW, $7 \mathrm{~Hz}$ to $12 \mathrm{~Hz}$ ) and a higher component in the beta frequency range (LATE HIGH, $13 \mathrm{~Hz}$ to $19 \mathrm{~Hz}$ ). The results are graphically summarized in Fig. 4 . The gray rectangles mark the TFROIs referring to the four effects described above that were then used to extract mean z-scores for comparisons across age groups and neuro-behavioral correlation analyses.

A direct comparison of YA and OA with regard to the four effects of interest revealed no reliable age differences in power modulations, all Fs $<1.75$ and all $p s>.19$, suggesting that overall similar inhibitory neuronal mechanisms related to alpha and beta oscillations contribute to the control of WM in both age groups.

To investigate the functionality of these mechanisms, we conducted neuro-behavioral correlation analyses between the neural effects and WM performance separately for the two age groups (see Fig. 5).

For young adults, the difference in oscillatory power between NREM and REM items was positively associated with WM performance during the late phases of stimulus processing in both low, LATE LOW: $r=.67$,
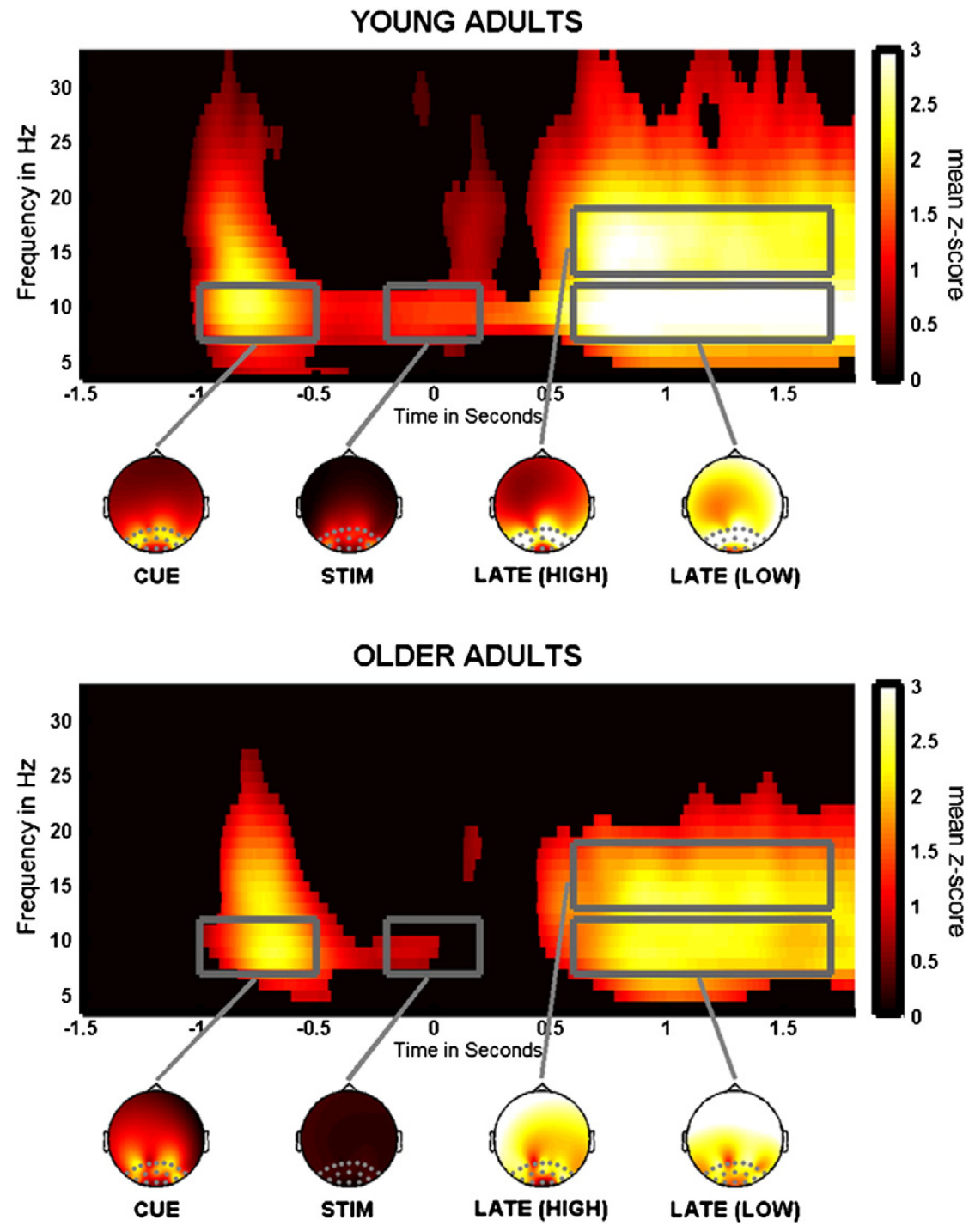

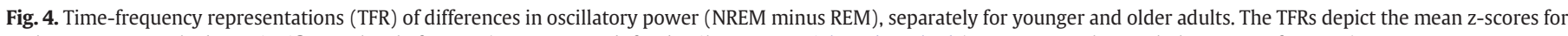

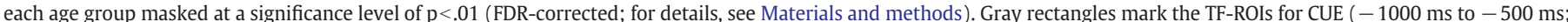

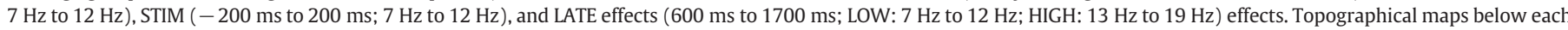

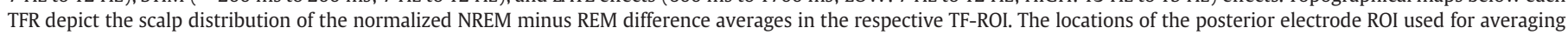
are highlighted in gray. 
$C_{\text {boot }}: 0.25-0.74, p<.05$, and high frequency ranges, LATE HIGH: $r=.51$, $C I_{\text {boot }}: 0.44-0.82, p<.05$. Neither during cue-processing, CUE: $r=.24$, $C I_{\text {boot }}:-0.25-0.61, p>.05$, nor during the early phases of stimulus processing, STIM: $r=.22, C_{\text {boot }}:-0.23-0.6, p>.05$, was the difference in oscillatory power predictive for WM performance in young adults.

Also among older adults, the differences in oscillatory alpha and beta power during later processing stages were predictive for interindividual differences in WM performance, LATE LOW: $r=.57, \mathrm{Cl}_{\text {boot }}$ : $0.31-0.77, p<.05$, LATE HIGH: $r=.58, C_{\text {boot }}: 0.25-0.8, p<.05$. In addition, the differential deployment of top-down control in anticipation of an upcoming WM item differentially contributed to the performance on the WM task in the group of older adults, as reflected in a significant correlation between WM performance and alpha power in the cue interval, CUE: $r=.55, C_{\text {boot }}: 0.22-0.78, p<.05$. Like in younger adults, the differences in oscillatory power during early phases of stimulus processing were not associated with inter-individual differences in WM performance, STIM: $r=.25, C_{\text {boot }}:-0.23-0.66, p>.05$.

We followed up on this analysis by taking into account the larger heterogeneity in the sample of older adults. Univariate ANOVAs with group (three levels: YA, OA-H, OA-L) as between subjects-factor were conducted separately for each effect of interest identified in the prior analysis (see Fig. 6). Early oscillatory markers of WM control (CUE, STIM) revealed comparable effects across the three groups, Fs $(2,39)<2.7, p s>.08$. However, for late time windows, the main effect of group reached significance, both $F s(2,39)>3.2$, $p s<.05$. Post-hoc contrasts revealed significantly smaller differences in oscillatory power (NREM - REM) at low and high frequency ranges for OA-L compared to YA (both $t>2.48$, both $p<.02$ ) and OA-H (both $t>2.42$, both $p<.02$ ), pointing to a specific impairment for low-performing $\mathrm{OA}$ in late selection processes. YA and $\mathrm{OA}-\mathrm{H}$ did not differ (both $t<1$, both $p>.75$ ). Thus, the analysis of mean-level differences between younger adults and the high- and low-performing older adults groups corroborated the important role of late inhibitory selection processes in controlling the contents of WM.

\subsection{Inter-trial phase stability}

With regard to inter-trial phase stability, we observed in both age groups a strong effect in response to the onset of the scene stimuli ( $0 \mathrm{~ms}$ to $400 \mathrm{~ms}$ ) with reliably stronger inter-trial phase locking for REM than for NREM items. This effect was most pronounced in lower frequency ranges ( $5 \mathrm{~Hz}$ to15 $\mathrm{Hz}$ ), with a slightly more widespread topography in older adults (see Fig. 7). The comparison of this effect between age groups revealed no significant age effect, $F<1.19$ and $p>28$, suggesting again that similar neuronal mechanisms contribute to the control of WM in both age groups.

To test the hypothesis of a functional contribution of increased phase stability to WM performance, we conducted neurobehavioral correlation analyses between the normalized differences in estimates of inter-trial phase-stability (REM minus NREM z-scores) and the percentage of correct responses in the WM task (see Fig. 8).

In young adults, the difference in inter-trial phase stability between REM and NREM items reliably predicted WM accuracy, $r=0.45, C_{\text {boot }}: 0.05-0.77, p<.05$. In older adults, the correlation was not significant, $r=0.12, C_{\text {boot }}:-0.33-0.66, p>.05$. However, when taking into account the presence of two separate groups of older adults (see Fig. 8, lower panels), we found a similar positive correlation of the difference in phase-stability with WM performance in high-performing older adults, $r=0.53, C_{\text {boot }}: 0.01-0.94, p<.05$. Only low-performing participants did not show a reliable correlation, $r=$

\section{YOUNG ADULTS}
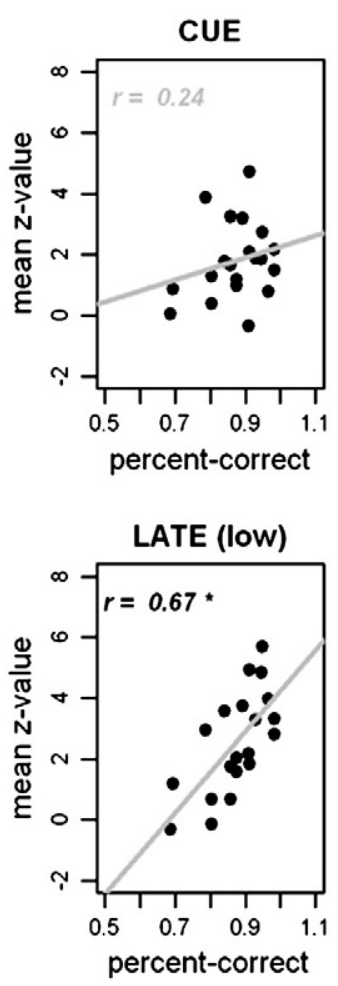
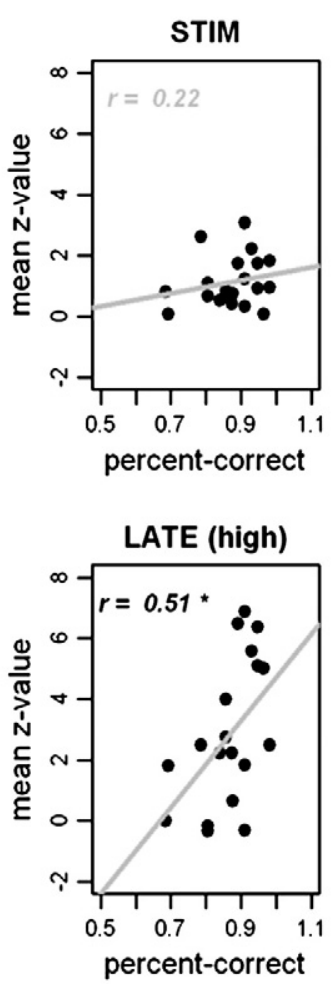

\section{OLDER ADULTS}
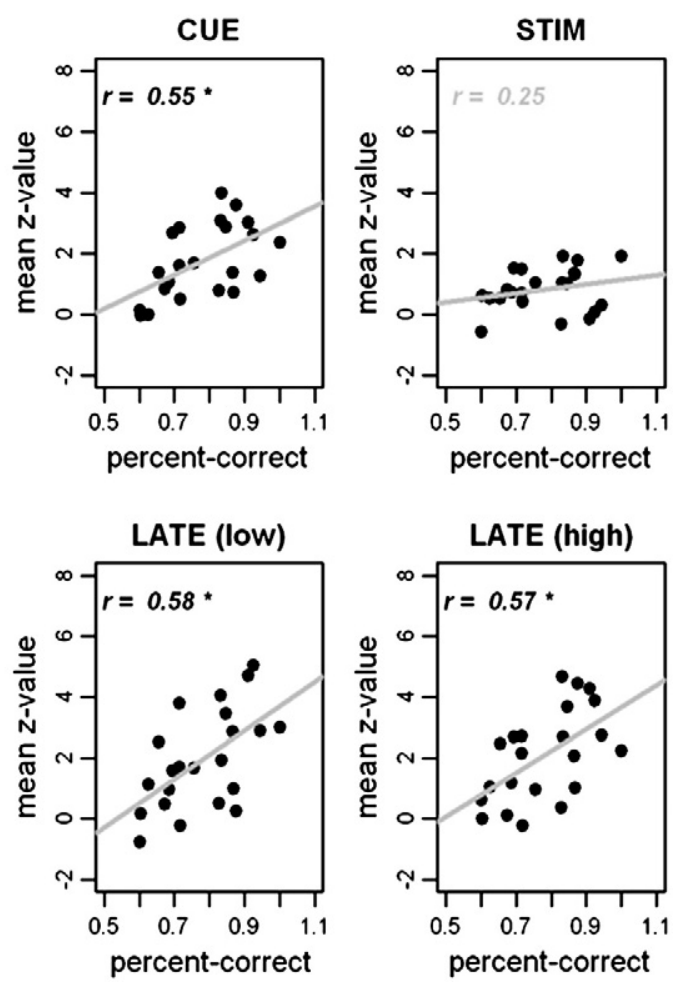

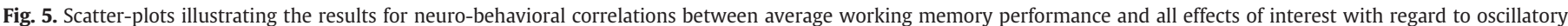

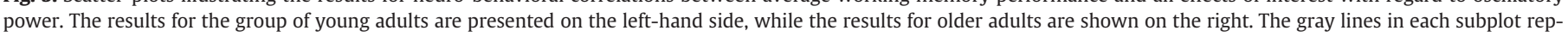

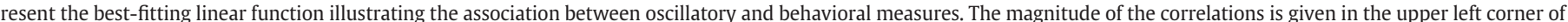

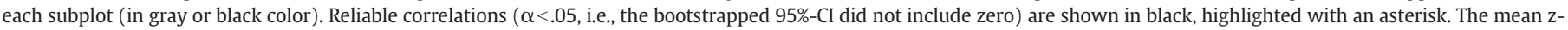

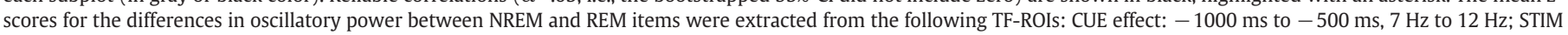
effect: $-200 \mathrm{~ms}$ to $200 \mathrm{~ms}, 7 \mathrm{~Hz}$ to $12 \mathrm{~Hz}$; LATE ALPHA: $600 \mathrm{~ms}$ to $1700 \mathrm{~ms}, 7 \mathrm{~Hz}$ to $12 \mathrm{~Hz}$; LATE BETA: $600 \mathrm{~ms}$ to $1700 \mathrm{~ms}, 13 \mathrm{~Hz}$ to $19 \mathrm{~Hz}$. 

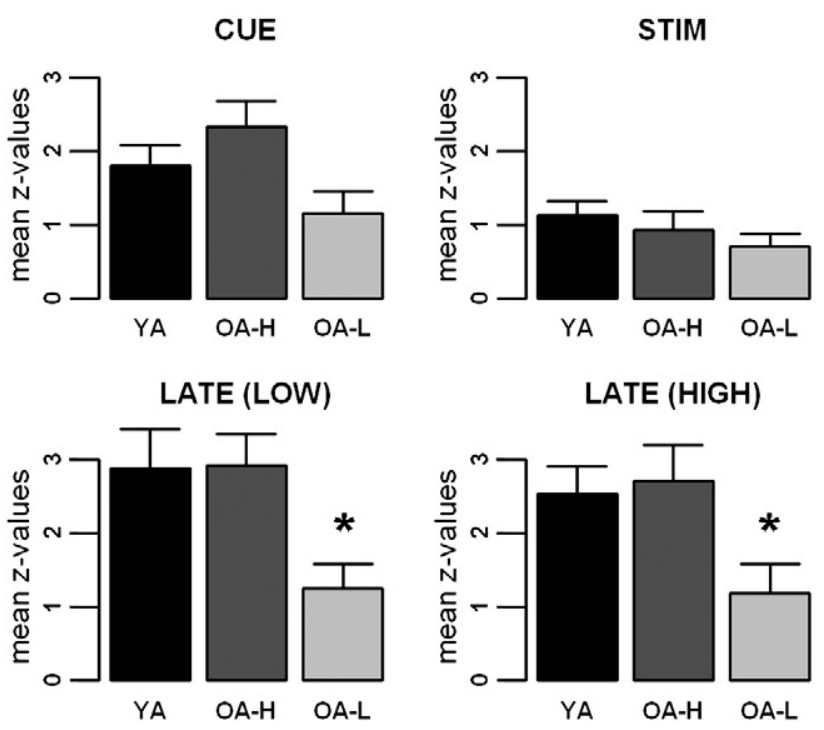

Fig. 6. Bar-charts representing group differences in power increases for NREM compared to REM items. Each bar depicts the mean z-score averaged within the TF-ROIs for the effects of interest: CUE, STIM, LATE (LOW), and LATE (HIGH). YA are depicted in black, high-performing OA (OA-H) in dark gray, and low-performing OA (OA-L) in light gray. The asterisks mark the reliably lower z-scores for OA-L in the late effect windows. Error bars denote the standard error of the mean.

$-0.41, C I_{\text {boot }}:-0.78-0.12, p>.05$, with a numerical trend in the opposite direction.

\section{Discussion}

The present study investigated whether non-phase locked and phase-locked oscillations contribute differentially to adult age differences in the processing of relevant and irrelevant items in WM (e.g., Freunberger et al., 2009). Younger and older participants were asked to remember REM items for later use while suppressing the processing of NREM items to prevent interference.

On the behavioral level, this differential processing of REM and NREM items led to a reliable memory advantage of REM over NREM items in a surprise recognition memory test in both age groups. Thus, participants from both age groups were clearly able to actively suppress the processing of NREM items and tried to efficiently encode the relevant information from REM items.

In addition to general age-differences in WM performance measures, we observed larger performance heterogeneity in the group of older adults. The increased heterogeneity of performance differences in groups of older adults has been mostly discussed on a theoretical level before (Lindenberger et al., 2008), and had only recently be considered as an influential factor for understanding neural correlates of attentional and memory functions (e.g., Duverne et al., 2009; Hedden et al., in press; Nagel et al., 2009; Nagel et al., 2011). We added to this evidence by reporting specific performance-dependent differences in oscillatory correlates of WM processes.

\subsection{Age and performance differences in control mechanism related to oscillatory amplitude}

In line with recent empirical evidence (e.g., Capotosto et al., 2009; Romei et al., 2008; Sauseng et al., 2009) and theoretical propositions (e.g., Jensen and Mazaheri, 2010; Klimesch et al., 2007), we regard the amplitude of oscillations in the alpha frequency range as an indicator of processes controlling the excitability of cortical regions.

Previous age comparative studies already reported overall reduced power as well as increasing phase-stability estimates in lower frequency ranges (e.g., delta/theta/alpha frequencies) with advancing age (e.g., Duffy et al., 1993; Muller et al., 2009), resonating with suggestions of impaired inhibitory control in older adults (Hasher and Zacks, 1988). However, general amplitude differences (see Supplemental figures) between age groups can hardly be interpreted unambiguously since they might be related also to age differences in brain size, brain geometry, or skull thickness (Frodl et al., 2001) and not to differences in cognitive processing per se. Accordingly, in the present report we did not focus on age main effects in the oscillatory EEG measures as they might reflect an accumulation of various influence not directly related to the task at hand (for similar arguments, see, Gazzaley and D'Esposito, 2005; Rugg and Morcom, 2005; Segalowitz and Davies, 2004; Werkle-Bergner et al., 2009). Here, we rather compared differences in task related phase and amplitude modulations and their contribution to WM performance in the different age groups, reflecting changes in the functionality of oscillatory mechanisms.

With regard to amplitude modulations induced by task affordances, we observed higher oscillatory power for NREM items compared to REM items during the cue, as well as early and late stimulus processing intervals in the alpha and beta frequency range in both younger and older adults. Increased oscillatory power for NREM compared to REM items reflects the amount of top-down control exerted during the different stages to suppress processing of NREM items (Freunberger et al., 2009; Jensen and Mazaheri, 2010; Klimesch et al., 2007; Romei et al., 2008). The observation of effect patterns common to both groups suggests that in principle the very same oscillatory mechanisms are present during neural information processing in younger and older adults and contribute to the control of WM contents. However, even when similar neuronal mechanisms are available, it is plausible that younger and older adults differentially recruit a subset of processes to support performance (e.g., Sander et al., 2011a; Velanova et al., 2007). We observed that in both age groups, differences in oscillatory power in late phases of stimulus processing were predictive for WM performance. The important role of late inhibitory selection processes in controlling the contents of WM was further corroborated by the observation that high and low performing older adults differed only in this late oscillatory marker. Thus, in contrast to results obtained by Gazzaley and co-workers (Gazzaley et al., 2008; Zanto et al., 2010), in our study the amplitude of alpha oscillations in response to cue and stimulus onset, which were conceived as markers of top-down control during early selection processes, did not discriminate between younger and older adults nor between high and low performing older adults. Rather, the comparison of high- and low-performing older adults points to late selection processes as the primary source of limitations in WM performance in the current study.

\subsection{Functional differences in phase-locked oscillations between younger and older adults}

We examined the temporal stability of oscillatory processes related to stimulus encoding by analyzing differences in phase stability between REM and NREM items. We expected that higher phase stability for relevant than for irrelevant information, as a signature of preferential encoding or feature binding, would predict WM accuracy (Hanslmayr et al., 2005; Mathewson et al., 2009). In line with this assumption, we observed positive correlations between increased phase stability for relevant compared to irrelevant information and WM performance in young adults. This result supports the hypothesis that phase synchronization improves the efficiency with which relevant information is encoded and processed (e.g., Freunberger et al., 2009; Fries, 2005; Hanslmayr et al., 2007; Klimesch et al., 2007, 2011; Mathewson et al., 2009). Phase synchronized neural oscillations may thereby gate information processing by providing time windows for efficient neural communication (Fries, 2005; Klimesch et al., 2007). 

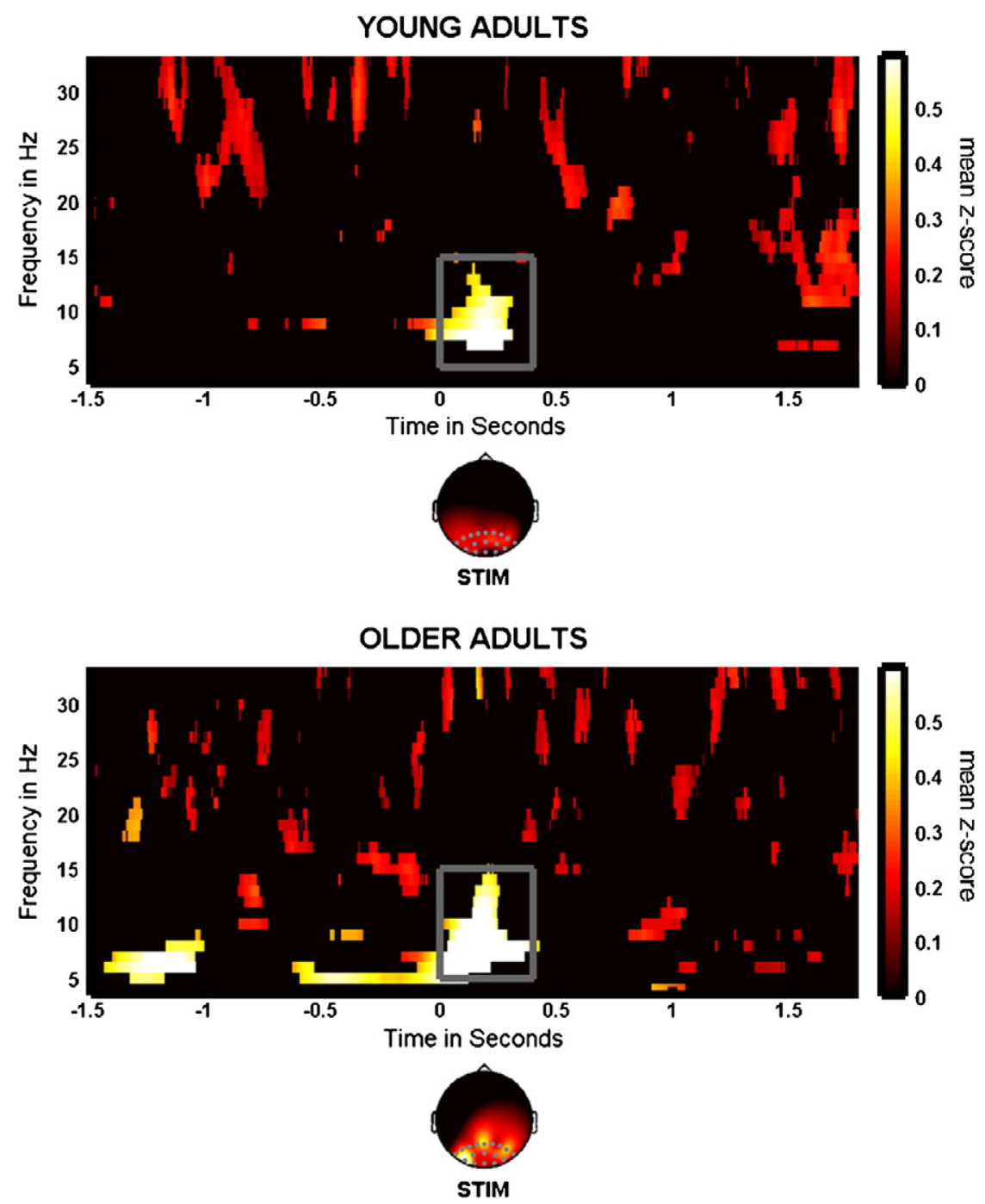

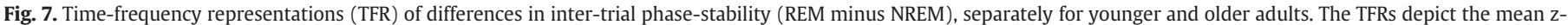

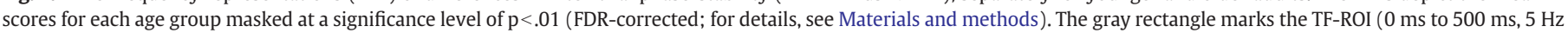

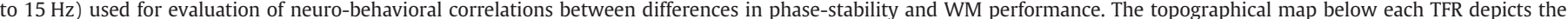

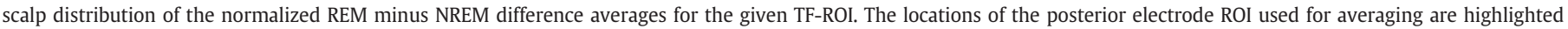
in gray.

In contrast, within the group of older adults as a whole, no association between phase stability for relevant information and WM performance was observed. Follow-up analyses revealed opposing correlative patterns in groups of low- and high-functioning older adults. Whereas younger adults and high-functioning older adults showed a positive association between phase stability and WM performance, low-functioning older adults did not profit from increased phase stability when encoding REM items. A similar age-differential association between inter-trial phase stability and cognitive processing was recently reported by Muller et al. (2009). In their study, relative to younger adults, older adults were characterized by higher phase-locking in response to auditory stimuli. Among older adults, the correlations between inter-trial phase-locking and independently assessed measures of perceptual speed were negative; in younger adults, the corresponding correlations were positive.

By analogy, we tentatively suggest that the inter-trial phase locking for the low-performing group of older adults in this study may reflect an unspecific form of stimulus-induced, bottom-up entrainment that does not differentiate between REM and NREM items (Gazzaley et al., 2005, 2008; Li et al., 2006; Park et al., 2004), perhaps reflecting senescent changes in the neurochemistry of the aging brain (Muller et al., 2009; Wang, 2010; Werkle-Bergner et al., 2009). In line with this conjectures, recent evidence from a lifespan study that relied on a change-detection WM task (Sander et al., 2012) reports higher inter-trial phase stability for older adults compared to children and younger adults that was interpreted as a loss of complexity of the neurophysiological response with advanced age (Cantero et al., 2009; Garrett et al., 2010; McIntosh et al., 2010; Sander et al., 2012; Thaler, 2002).

\subsection{Performance related differences in the control of WM-contents among older adults}

It is important to note that the group of high-performing older adults did not differ from the group of younger adults in its ability to extract relevant information and to exert control during any stage of stimulus processing. This applies to inhibitory mechanisms reflected in amplitude modulations as well as early encoding processes related to stimulus specific modulations of inter-trial phasestability. By contrast, low-performing older adults were specifically characterized by smaller differences in oscillatory power between REM and NREM items. Furthermore, encoding processes related to synchronized oscillatory mechanisms appear less efficient in lowperforming older participants. 

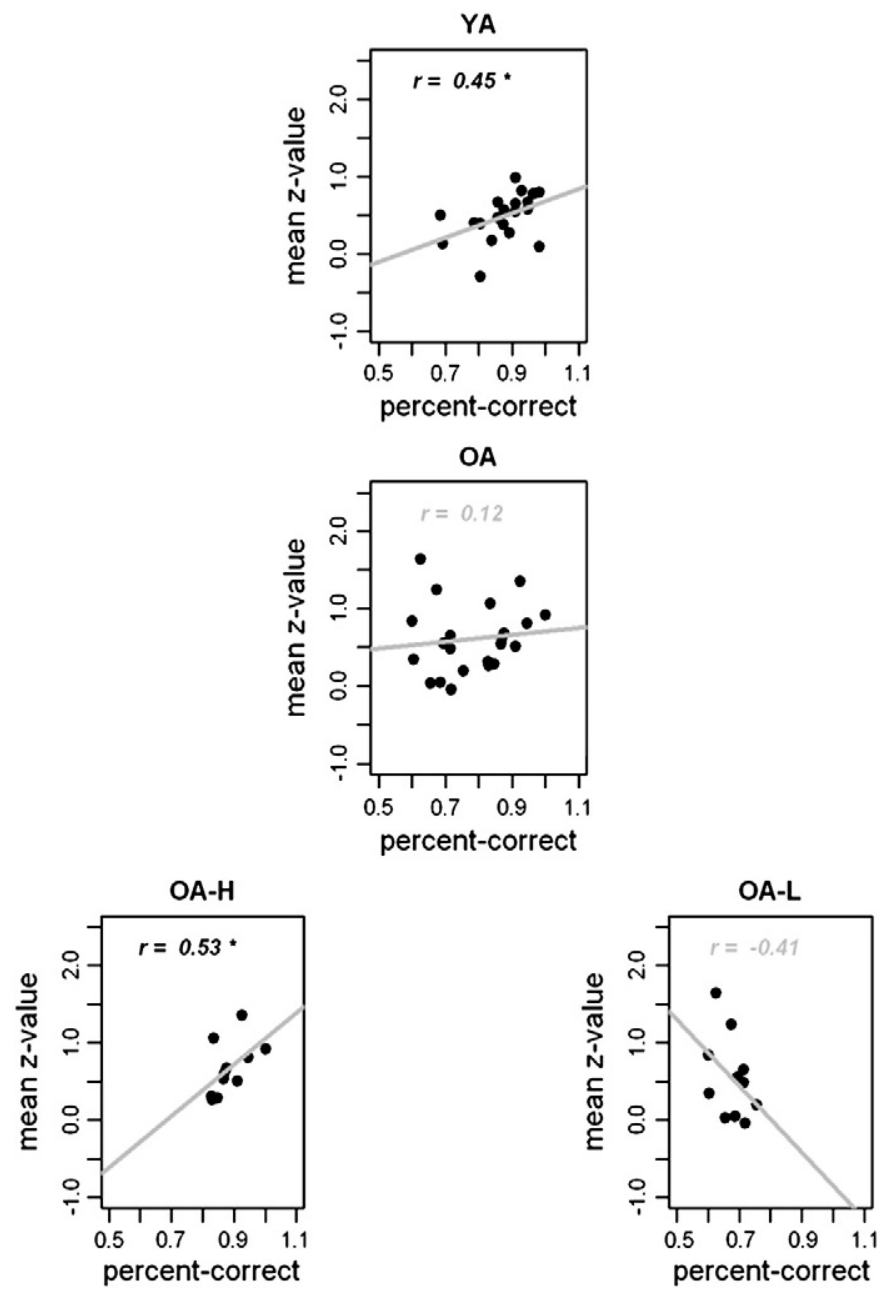

Fig. 8. Scatter-plots illustrating the results for neuro-behavioral correlations between average working memory performance and differences in phase-stability between REM and NREM items. The upper and middle panels show the results for the groups of YA and OA, respectively. The bottom panels summarize the results for high $(\mathrm{OA}-\mathrm{H})$ and low performing older adults (OA-L) on the left and right, respectively. Each black circle represents a single participant. The gray lines in each subplot represent the best-fitting linear function illustrating the association between oscillatory and behavioral measures. The magnitude of the correlations is given in the upper left corner of each subplot (in gray or black color). Reliable correlations ( $\alpha<.05$, i.e., the bootstrapped 95\%-CI did not include zero) are shown in black, highlighted with an asterisk. The z-scores for the difference between REM and NREM items in estimates of inter-trial phase stability were extracted from the following TF-ROI: $0 \mathrm{~ms}$ to $500 \mathrm{~ms}, 5 \mathrm{~Hz}$ to $15 \mathrm{~Hz}$.

These results are in line with the load-shift model of executive functioning recently proposed by Velanova et al. (2007). According to this view, younger adults as well as high-performing older adults may have relied on a combination of early- and late-selection processes with considerable resources expended at early stages to constrain processing of relevant information. Due to senescent decline in top-down control mechanisms, i.e., related to frontal-striatal systems (e.g., Backman et al., 2006; Raz et al., 2005), the lowperforming group of older adults may have failed to differentially adjust the processing of REM and NREM items early on, resulting in poorly constrained representations entering later processing stages (Li et al., 2006), which increases the demands on late selection mechanisms. But, shifting demands between early and late selection operations appears limited. When individual performance limits are reached, oscillatory mechanisms of cortical excitability regulation in the service of differentiating between relevant and irrelevant information break down (Sander et al., 2012).

But what may lead to the inability of low-performing older adults to adjust control processes during encoding of WM contents? Only a few studies investigated the oscillatory mechanisms related to WM performance in older adults so far and little is known about how lifespan changes in neuroanatomy (e.g., Raz et al., 2005) and neurochemistry (e.g., Backman et al., 2006; Li et al., 2006) during maturation, learning, and senescence affect synchronization properties on a network level as they are reflected in phase and amplitude measures of scalp EEG recordings (e.g., Muller et al., 2009; Werkle-Bergner et al., 2009). Nevertheless, speculative hints on the possible neuroanatomical difference may be derived from fMRI findings. In line with the present report, recent investigations of age-differences in fMRI activation patterns during WM-tasks demonstrate a dependence of the ability to modulate neuro-vascular responses on the individual performance level (e.g., Nagel et al., 2009, 2011; Schneider-Garces et al., 2010). In addition, Hedden et al. (in press) could link a failure to modulate fMRI responses according to attentional demands in a subset of their older participants to elevated white matter hyperintensity (WMH) burden. This finding is of special interest in the present context, as WMHs were also shown to affect oscillatory EEG patterns (Babiloni et al., 2008a, 2009), especially related to fronto-parietal networks (Babiloni et al., 2008b). It is tempting to speculate that interindividual differences in white matter integrity may be commonly related to the ability to modulate attentional resources as reflected in fMRI activation patterns and oscillatory EEG markers. Future research, preferably with multi-modal imaging approaches (e.g., Debener and Herrmann, 2008; Debener et al., 2006; Eichele et al., 2009), is definitely warranted to foster insights into dynamic structure-function relationships across the adult lifespan.

\subsection{Conclusion}

Taken together, our data are consistent with the propositions (Freunberger et al., 2011) that (a) the greater inter-trial phase stability for REM relative to NREM items reflects the preferential encoding or binding of REM items; (b) the higher power of oscillations around the alpha frequency range for NREM relative to REM items reflects the inhibitory top-down suppression of task-irrelevant information. Dissociable patterns of phase-locked and non-phase locked EEG oscillations revealed age-group and performance differences in component processes of WM. Specifically, low-level binding mechanisms as reflected in measures of inter-trial phase stability were shown to operate less effectively in low-performing older adults. In addition, non-phase locked activity reflecting the suppression of irrelevant information was also impaired in this group. Similar to other recent studies (Duverne et al., 2009; Nagel et al., 2009, 2011; Schneider-Garces et al., 2010), the present results underscore the heterogeneity of WM and episodic memory functioning among older adults, and are consistent with the proposition that older adults with more "youth-like" processing patterns tend to show higher levels of performance (e.g., Nagel et al., 2009). The present results are in line with a recently proposed conceptual framework for studying lifespan changes in episodic (Shing et al., 2008, 2009; Werkle-Bergner et al., 2006) and working memory (Sander et al., 2011a, 2011b), that dissociates the contributions of low-level feature binding and (strategic) control components to adult age differences in memory performance.

\section{Acknowledgements}

This study was conducted within the project "Cognitive and Neuronal Dynamics of Memory across the Lifespan (CONMEM)' at the Center for Lifespan Psychology, Max Planck Institute for Human Development. The research was partially financed by the Max Planck Society. MW-B's work is supported by a grant from the German Research Foundation (DFG, HE 3347/3-1).

We gratefully thank the student and technical assistants of the CONMEM project for their support during data collection and the 
participants for their cooperation. We give special thanks to Yana Fandakova, Paul Sauseng, and Yee Lee Shing for valuable discussions.

\section{Appendix A. Supplementary data}

Supplementary data to this article can be found online at doi:10. 1016/j.neuroimage.2011.11.071.

\section{References}

Babiloni, C., Frisoni, G.B., Pievani, M., Toscano, L., Del Percio, C., Geroldi, C., Eusebi, F., Miniussi, C., Rossini, P.M., 2008a. White-matter vascular lesions correlate with alpha EEC sources in mild cognitive impairment. Neuropsychologia 46, 1707-1720.

Babiloni, C., Frisoni, G.B., Pievani, M., Vecchio, F., Infarinato, F., Geroldi, C., Salinari, S. Ferri, R., Fracassi, C., Eusebi, F., Rossini, P.M., 2008b. White matter vascular lesion are related to parietal-to-frontal coupling of EEG rhythms in mild cognitive impairment. Hum. Brain Mapp. 29, 1355-1367.

Babiloni, C., Pievani, M., Vecchio, F., Geroldi, C., Eusebi, F., Fracassi, C., Fletcher, E., De Carli, C., Boccardi, M. Rossini, P.M., Frisoni, G.B, 2009. White-matter lesions along the cholinergic tracts are related to cortical sources of EEG rhythms in amnesic mild cognitive impairment. Hum. Brain Mapp. 30, 1431-1443.

Backman, L., Nyberg, L., Lindenberger, U., Li, S.C., Farde, L., 2006. The correlative triad among aging, dopamine, and cognition: current status and future prospects. Neurosci. Biobehav. Rev. 30, 791-807.

Baltes, P.B., Lindenberger, U., 1997. Emergence of a powerful connection between sensory and cognitive functions across the adult life span: a new window to the study of cognitive aging? Psychol. Aging 12, 12-21.

Bauer, M., Oostenveld, R., Peeters, M., Fries, P., 2006. Tactile spatial attention enhances gamma-band activity in somatosensory cortex and reduces low-frequency activity in parieto-occipital areas. J. Neurosci. 26, 490-501.

Benjamini, Y., Hochberg, Y., 1995. Controlling the false discovery rate: a practical and powerful approach to multiple testing. J. R. Stat. Soc. B Methodol. 57, 289-300.

Cantero, J.L., Atienza, M., Cruz-Vadell, A., Suarez-Gonzalez, A., Gil-Neciga, E., 2009. Increased synchronization and decreased neural complexity underlie thalamocortical oscillatory dynamics in mild cognitive impairment. Neuroimage 46, 938-948.

Capotosto, P., Babiloni, C., Romani, G.L., Corbetta, M., 2009. Frontoparietal cortex controls spatial attention through modulation of anticipatory alpha rhythms. J. Neurosci. 29, 5863-5872.

Cohen, J., 1988. Statistical Power Analysis for the Behavioral Sciences, 2nd ed. Lawrence Erlbaum Associates, Hillsdale.

Cowan, N., Naveh-Benjamin, M., Kilb, A., Saults, J.S., 2006. Life-span development of visual working memory: when is feature binding difficult. Dev. Psychol. 42, 1089-1102.

Debener, S., Herrmann, C.S., 2008. Integration of EEG and fMRI (Editorial) Int. J. Psychophysiol. 67, 159-160.

Debener, S., Ullsperger, M., Siegel, M., Engel, A.K., 2006. Single-trial EEG-fMRI reveals the dynamics of cognitive function. Trends Cogn. Sci. 10, 558-563.

Delorme, A., Sejnowski, T., Makeig, S., 2007. Enhanced detection of artifacts in EEG data using higher-order statistics and independent component analysis. Neuroimage 34, 1443-1449.

Duffy, F.H., McAnulty, G.B., Albert, M.S., 1993. The pattern of age-related differences in electrophysiological activity of healthy males and females. Neurobiol. Aging 14, 73-84.

Duverne, S., Motamedinia, S., Rugg, M.D., 2009. The relationship between aging, performance, and the neural correlates of successful memory encoding. Cereb. Cortex 19 733-744

Eichele, T., Calhoun, V.D., Debener, S., 2009. Mining EEG-fMRI using independent component analysis. Int. J. Psychophysiol. 73, 53-61.

Eng, H.Y., Chen, D., Jiang, Y., 2005. Visual working memory for simple and complex visual stimuli. Psychon. Bull. Rev. 12, 1127-1133.

Fei-Fei, L., Perona, P., 2005. A Bayesian hierarchical model for learning natural scene categories. 2005 IEEE Computer Society Conference on Computer Vision and Pattern Recognition, vol 2, pp. 524-531 (Proceedings) (1216).

Freunberger, R., Fellinger, R., Sauseng, P., Gruber, W., Klimesch, W., 2009. Dissociation between phase-locked and nonphase-locked alpha oscillations in a working memory task. Hum. Brain Mapp. 30, 3417-3425.

Freunberger, R., Werkle-Bergner, M., Griesmayr, B., Lindenberger, U., Klimesch, W., 2011. Brain oscillatory correlates of working memory constraints. Brain Res, $1375,93-102$.

Fries, P., 2005. A mechanism for cognitive dynamics: neuronal communication through neuronal coherence. Trends Cogn. Sci. 9, 474-480.

Frodl, T., Meisenzahl, E.M., Mueller, D., Leinsinger, G., Juckel, G., Hahn, K., Moeller, H.-J. Hegerl, U., 2001. The effect of the skull on event-related P300. Clin. Neurophysiol. $112,1773-1776$.

Garrett, D.D., Kovacevic, N., McIntosh, A.R., Grady, C.L., 2010. Blood oxygen leveldependent signal variability is more than just noise. J. Neurosci. 30, 4914-4921.

Gazzaley, A., Clapp, W., Kelley, J., McEvoy, K., Knight, R.T., D'Esposito, M., 2008. Age-related top-down suppression deficit in the early stages of cortical visual memory processing. Proc. Natl. Acad. Sci. U. S. A. 105, 13122-13126.

Gazzaley, A., Cooney, J.W., Rissman, J., D'Esposito, M., 2005. Top-down suppression deficit underlies working memory impairment in normal aging. Nat. Neurosci. 8, 1298-1300.

Gazzaley, A., D'Esposito, M., 2005. BOLD functional MRI and cognitive aging. In: Cabeza R., Nyberg, L., Park, D. (Eds.), Cognitive Neuroscienceof Aging: Linking Cognitive and Cerebral Aging. Oxford University Press, Oxford.
Geigy, J.R., 1977. Wissenschaftliche Tabellen (Scientific Tables). J.R. Geigy AG, Basel, Switzerland.

Hanslmayr, S., Aslan, A., Staudigl, T., Klimesch, W., Herrmann, C.S., Bauml, K.H., 2007. Prestimulus oscillations predict visual perception performance between and within subjects. Neuroimage 37, 1465-1473.

Hanslmayr, S., Klimesch, W., Sauseng, P., Gruber, W., Doppelmayr, M., Freunberger, R., Pecherstorfer, T., 2005. Visual discrimination performance is related to decreased alpha amplitude but increased phase locking. Neurosci. Lett. 375, 64-68.

Hartigan, J.A., Hartigan, P.M., 1985. The dip test of unimodality. Ann. Stat. 13, 70-84.

Hasher, L., Zacks, R.T., 1988. Working memory, comprehension, and aging: a review and a new view. In: Bower, G.H. (Ed.), The Psychology of Learning and Motivation. Academic Press, New York, pp. 193-225.

Hedden, T., Van Dijk, K.R., Shire, E.H., Sperling, R.A., Johnson, K.A., Buckner, R.L., in press. Failure to modulate attentional control in advanced aging linked to white matter pathology. Cereb Cortex.

Holmes, A., Friston, K., 1998. Generalisability, random effects, and population inference. Neuroimage 7, S754

Hughett, P., 2007. Accurate computation of the F-to-z and t-to-z transforms for large arguments journal of statistical software. Code Snippets 23, 1-5.

Hullett, C., Levine, T., 2003. The overestimation of effect sizes from F values in metaanalysis: the cause and a solution. Commun. Monogr. 70, 52-67.

Jensen, O., Mazaheri, A., 2010. Shaping functional architecture by oscillatory alpha activity: gating by inhibition. Front. Hum. Neurosci. 4, 186.

Jost, K., Bryck, R.L., Vogel, E.K., Mayr, U., 2011. Are old adults just like low working memory young adults? Filtering efficiency and age differences in visual working memory. Cereb. Cortex 21, 1147-1154.

Jung, T.P., Makeig, S., Humphries, C., Lee, T.W., McKeown, M.J., Iragui, V., Sejnowski, T.J., 2000. Removing electroencephalographic artifacts by blind source separation. Psychophysiology 37, 163-178.

Klimesch, W., Fellinger, R., Freunberger, R., 2011. Alpha oscillations and early stages of visual encoding. Front. Psychol. 2, 118

Klimesch, W., Sauseng, P., Hanslmayr, S., 2007. EEG alpha oscillations: the inhibitiontiming hypothesis. Brain Res. Rev. 53, 63-88.

Lachaux, J.P., Rodriguez, E., Martinerie, J., Varela, F.J., 1999. Measuring phase synchrony in brain signals. Hum. Brain Mapp. 8, 194-208.

Lehrl, S., 1977. Mehrfachwahl-Wortschatz-Test B (Multiple-choice Knowledge Test-B (MWT-B)). Straube, Erlangen, Germany.

Li, S.C., Brehmer, Y., Shing, Y.L., Werkle-Bergner, M., Lindenberger, U., 2006. Neuromodulation of associative and organizational plasticity across the life span: empirical evidence and neurocomputational modeling. Neurosci. Biobehav. Rev. 30, 775-790.

Lindenberger, U., Mayr, U., Kliegl, R., 1993. Speed and intelligence in old age. Psychol. Aging 8, 207-220.

Lindenberger, U., Nagel, I.E., Chicherio, C., Li, S.C., Heekeren, H.R., Backman, L, 2008. Age-related decline in brain resources modulates genetic effects on cognitive functioning. Front. Neurosci. 2, 234-244

Luck, S.J., Vogel, E.K., 1997. The capacity of visual working memory for features and conjunctions. Nature 390, 279-281.

Mathewson, K.E., Gratton, G., Fabiani, M., Beck, D.M., Ro, T., 2009. To see or not to see: prestimulus alpha phase predicts visual awareness. J. Neurosci. 29, 2725-2732.

McIntosh, A.R., Kovacevic, N., Lippe, S., Garrett, D., Grady, C., Jirsa, V., 2010. The development of a noisy brain. Arch. Ital. Biol. 148, 323-337.

Miller, G.A., 1956. The magical number seven, plus or minus two:some limits on our capacity for processing information. Psych. Rev. 63

Muller, V., Gruber, W., Klimesch, W., Lindenberger, U., 2009. Lifespan differences in cortical dynamics of auditory perception. Dev. Sci. 12, 839-853.

Mumford, J.A., Poldrack, R.A., 2007. Modeling group fMRI data. Soc. Cogn. Affect. Neurosci. 2, 251-257.

Nagel, I.E., Preuschhof, C., Li, S.C., Nyberg, L., Backman, L., Lindenberger, U., Heekeren, H.R., 2009. Performance level modulates adult age differences in brain activation during spatial working memory. Proc. Natl. Acad. Sci. U. S. A. 106, 22552-22557.

Nagel, I.E. Preuschhof, C. Li, S.C., Nyberg, L., Backman, L, Lindenberger, U., Heekeren, H.R., 2011. Load modulation of BOLD response and connectivity predicts working memory performance in younger and older adults. J. Cogn. Neurosci. 23, 2030-2045.

Nichols, T., Hayasaka, S., 2003. Controlling the familywise error rate in functional neuroimaging: a comparative review. Stat. Methods Med. Res. 12, 419-446.

Oldfield, R.C., 1971. The assessment and analysis of handedness: the Edinburgh inventory. Neuropsychologia 9, 97-113.

Palva, S., Palva, J.M., 2007. New vistas for $\alpha$-frequency band oscillations. Trends Neurosci. 30, 150-158.

Park, D.C. Payer, D, 2006. Working memory across the adult lifespan. lifespan cognition: mechanisms of change. In: Bialystok, E.E.C., Fergus, I.M. (Eds.), Lifespan Cognition: Mechanisms of Change. Oxford University Press, New York, NY, US, pp. 128-142 (7 pp.).

Park, D.C., Polk, T.A., Park, R., Minear, M., Savage, A., Smith, M.R., 2004. Aging reduces neural specialization in ventral visual cortex. Proc. Natl. Acad. Sci. U. S. A. 101, 13091-13095.

Philiastides, M.G., Sajda, P., 2006. Temporal characterization of the neural correlates of perceptual decision making in the human brain. Cereb. Cortex 16, 509-518.

Raz, N., Lindenberger, U., Rodrigue, K.M., Kennedy, K.M., Head, D., Williamson, A., Dahle, C., Gerstorf, D., Acker, J.D., 2005. Regional brain changes in aging healthy adults: general trends, individual differences and modifiers. Cereb. Cortex 15, 1676-1689.

Romei, V., Brodbeck, V., Michel, C., Amedi, A., Pascual-Leone, A., Thut, G., 2008. Spontaneous fluctuations in posterior alpha-band EEG activity reflect variability in excitability of human visual areas. Cereb. Cortex 18, 2010-2018.

Rugg, M.D., Morcom, A.M., 2005. The relationship between brain activity, cognitive performance, and aging: the case of memory. In: Cabeza, R., Nyberg, L., Park, D. (Eds.), 
Cognitive Neuroscience of Aging: Linking Cognitive and Cerebral Aging. Oxford University Press, New York.

Salthouse, T.A., 1996. The processing-speed theory of adult age differences in cognition. Psychol. Rev. 103, 403-428.

Sander, M.C., Werkle-Bergner, M., Lindenberger, U., 2011a. Binding and strategic selection in working memory: a lifespan dissociation. Psychol. Aging 26, 612-624.

Sander, M.C., Werkle-Bergner, M., Lindenberger, U., 2011b. Contralateral delay activity reveals life-span age differences in top -down modulation of working memory contents. Cereb. Cortex. 21, 2809-2819.

Sander, M.C., Werkle-Bergner, M., Lindenberger, U., 2012. Amplitude modulations and inter-trial phase stability of alpha-oscillations differentially reflect working memory constraints across the lifespan. Neuroimage 59, 646-654.

Sauseng, P., Klimesch, W., Heise, K.F., Gruber, W.R., Holz, E., Karim, A.A., Glennon, M., Gerloff, C., Birbaumer, N., Hummel, F.C., 2009. Brain oscillatory substrates of visual short-term memory capacity. Curr. Biol. 19, 1846-1852.

Schneider-Garces, N.J., Gordon, B.A., Brumback-Peltz, C.R., Shin, E., Lee, Y., Sutton, B.P., Maclin, E.L., Gratton, G., Fabiani, M., 2010. Span, CRUNCH, and beyond: working memory capacity and the aging brain. J. Cogn. Neurosci. 22, 655-669.

Segalowitz, S.J., Davies, P.L., 2004. Charting the maturation of the frontal lobe: an electrophysiological strategy. Brain Cogn. 55, 116-133.

Shing, Y.L., Werkle-Bergner, M., Brehmer, Y., Muller, V., Li, S.C., Lindenberger, U., 2009. Episodic memory across the lifespan: the contributions of associative and strategic components. Neurosci. Biobehav. Rev.

Shing, Y.L., Werkle-Bergner, M., Li, S.C., Lindenberger, U., 2008. Associative and strategic components of episodic memory: a life-span dissociation. J. Exp. Psychol. Gen. 137, 495-513.

Singer, W., Gray, C.M., 1995. Visual feature integration and the temporal correlation hypothesis. Annu. Rev. Neurosci. 18, 555-586.

Snodgrass, J.G., Corwin, J., 1988. Pragmatics of measuring recognition memory-applications to dementia and amnesia. J. Exp. Psychol. Gen. 117, 34-50.

Sternberg, S., 1966. High-speed scanning in human memory. Science 153, 652-654.
Tallon-Baudry, C., Bertrand, O., Peronnet, F., Pernier, J., 1998. Induced gamma-band activity during the delay of a visual short-term memory task in humans. J. Neurosci. 18, 4244-4254.

Thaler, D.S., 2002. Design for an aging brain. Neurobiol. Aging 23, 13-15.

Thut, G., Nietzel, A., Brandt, S.A., Pascual-Leone, A., 2006. Alpha-band electroencephalographic activity over occipital cortex indexes visuospatial attention bias and predicts visual target detection. J. Neurosci. 26, 9494-9502.

van Dijk, H., van der Werf, J., Mazaheri, A., Medendorp, W.P., Jensen, O., 2010. Modulations in oscillatory activity with amplitude asymmetry can produce cognitively relevant event-related responses. Proc. Natl. Acad. Sci. U. S. A. 107, 900-905.

Velanova, K., Lustig, C., Jacoby, L.L., Buckner, R.L., 2007. Evidence for frontally mediated controlled processing differences in older adults. Cereb. Cortex 17, 1033-1046.

Vogel, E.K., Awh, E., 2008. How to exploit diversity for scientific gain: using individual differences to constrain cognitive theory. Curr. Dir. Psychol. Sci. 17, 171-176.

Vogel, E.K., McCollough, A.W., Machizawa, M.G., 2005. Neural measures reveal individual differences in controlling access to working memory. Nature 438, 500-503.

Von der Malsburg, C., 1981. The Correlation Theory of Brain Function. Max Planck Institute for Biophysical Chemistry, Göttingen.

Wang, X.J., 2010. Neurophysiological and computational principles of cortical rhythms in cognition. Physiol. Rev. 90, 1195-1268.

Wechsler, D., 1958. The Measurement and Appraisal of Adult Intelligence, 4th ed. Williams \& Wilkins, Baltimore.

Werkle-Bergner, M., Muller, V., Li, S.C., Lindenberger, U., 2006. Cortical EEG correlates of successful memory encoding: implications for lifespan comparisons. Neurosci. Biobehav. Rev. 30, 839-854.

Werkle-Bergner, M., Shing, Y.L., Muller, V., Li, S.C., Lindenberger, U., 2009. EEG gammaband synchronization in visual coding from childhood to old age: evidence from evoked power and inter-trial phase locking. Clin. Neurophysiol. 120, 1291-1302.

Zanto, T.P., Toy, B., Gazzaley, A., 2010. Delays in neural processing during working memory encoding in normal aging. Neuropsychologia 48, 13-25. 